\title{
Modelagem Baseada no Indivíduo para Análise da Dinâmica Espaço-Temporal do HLB do Citrus
}

\author{
Raphael G. d'A. Vilamiu \\ CEFET-RJ, Campus Angra dos Reis \\ 23953-030, Angra dos Reis, RJ \\ E-mail: vilamiu@gmail.com \\ Sônia Ternes \\ Embrapa Informática \\ 13083-886, Campinas, SP \\ E-mail: sonia.ternes@embrapa.br
}

\begin{abstract}
Resumo: O Huanglongbing (HLB) é uma séria doença incurável, que traz grande prejuízo aos citricultores. No Brasil, o psilídeo Diaphorini citri é o principal inseto-vetor, o qual adquire a bactéria causadora do HLB ao se alimentar de plantas infectadas. Este trabalho apresenta um modelo baseado no indivíduo para analisar a dinâmica espaço-temporal da transmissão do HLB em um pomar de citros. As simulações indicam que a simples remoção de plantas contaminadas sintomáticas é uma prática insuficiente para controle da doença.
\end{abstract}

\section{Introdução}

A citricultura é uma atividade de grande importância para o Brasil. O país é o maior produtor mundial de citros, tendo obtido uma safra de 21 milhões de toneladas em 2011 [1], negócio que gera 4,5 bilhões de dólares por ano e é responsável por cerca de 400 mil empregos diretos e indiretos [2]. Projeções oficiais [3] indicam que a safra brasileira deve alcançar 23,8 milhões de toneladas em 2023, o que equivale a uma taxa anual de crescimento de $1,7 \%$.

A doença conhecida como Huanglongbing (HLB), identificada em 2004 no Estado de São Paulo, maior região produtora brasileira, é atualmente a grande preocupação entre os produtores de citros. No Brasil, o HLB é transmitido pelo psilídeo Diaphorina citri, que adquire a bactéria (Candidatus Liberibacter) ao se alimentar de plantas infectadas [4]. O HLB é incurável, afeta todas as variedades de citros e reduz a produtividade no campo. A doença possui um longo período de incubação, durante o qual as plantas não apresentam sintomas, mas são infecciosas. A principal estratégia para o combate à doença é a remoção periódica de plantas sintomáticas e o controle da população de psilídeos [4].

Modelos matemáticos vem sendo desenvolvidos para auxiliar no entendimento da dinâmica da doença [5,6,7]. Vilamiu et al [8] desenvolveram um modelo matemático determinístico para representar a dinâmica de infecção do HLB em plantações de citros, incluindo retardo no tempo para o período de incubação da doença nas plantas e para o período de estágio de ninfa da Diaphorina citri.

Além dos modelos matemáticos clássicos, a modelagem baseada no indivíduo (MBI) tem sido usada na análise quantitativa de processos ecológicos [9], pois é adequada para modelar populações heterogêneas, e facilita o estudo da dinâmica espacial, em contraposição aos modelos determinísticos. Nessa abordagem, a população é composta por um conjunto de entidades discretas (indivíduos) e autônomas. A ênfase do MBI está na interação entre os indivíduos, que carregam tantas informações quanto forem necessárias para detalhar o modelo, 
e cujas características evoluem estocasticamente no tempo. A dinâmica do sistema é determinada por regras a serem aplicadas a cada indivíduo.

O objetivo deste trabalho é desenvolver um modelo baseado no indivíduo para representar a dinâmica espaço-temporal de transmissão do HLB pelo seu inseto vetor $D$. citri, considerando as premissas do modelo determinístico e parâmetros biológicos usados em Vilamiu et al [8].

\section{Modelo Baseado no Indivíduo}

O MBI foi formulado utilizando premissas epidemiológicas correspondentes ao trabalho de Vilamiu et al. [8], considerando a metodologia apresentada em Nepomuceno [10]. A Tabela 1 descreve as três populações consideradas no modelo e as correspondentes características atribuídas a cada indivíduo da população.

Tabela 1: Populações e características dos indivíduos considerados no MBI

\begin{tabular}{|c|c|c|}
\hline População & Característica & Categoria \\
\hline \multirow{5}{*}{$\begin{array}{l}\text { H: } \\
\text { Hospedeiro }\end{array}$} & \multirow{4}{*}{$\begin{array}{l}\mathrm{H}_{1} \text { : estado } \\
\text { epidemiológico }\end{array}$} & 0 : suscetível \\
\hline & & $\begin{array}{l}0<\mathrm{x}<\tau_{\mathrm{h}} \text { : infectado assintomático (incubação), com x igual ao } \\
\text { número de dias no período de incubação }\end{array}$ \\
\hline & & $\mathrm{x}=\tau_{\mathrm{h}}:$ infectado sintomático \\
\hline & & -1 : removido \\
\hline & $\begin{array}{l}\mathrm{H}_{2} \text { : localização } \\
\text { geográfica }\end{array}$ & $\left(\mathrm{x}_{\mathrm{h}}, \mathrm{y}_{\mathrm{h}}\right)$ : localização na área de plantio \\
\hline \multirow{4}{*}{$\begin{array}{l}\mathrm{N} \text { : inseto } \\
\text { vetor em } \\
\text { estágio de } \\
\text { ninfa }\end{array}$} & \multirow{2}{*}{$\begin{array}{l}\mathrm{N}_{1} \text { : estado } \\
\text { epidemiológico }\end{array}$} & 0: não infectivo \\
\hline & & 1: infectivo \\
\hline & $\begin{array}{l}\mathrm{N}_{2} \text { : localização } \\
\text { geográfica }\end{array}$ & $\left(\mathrm{x}_{\mathrm{n}}, \mathrm{y}_{\mathrm{n}}\right)$ : localização na área de plantio (sobre uma planta) \\
\hline & $\mathrm{N}_{3}$ : idade & Número de dias na fase de ninfa \\
\hline \multirow{4}{*}{$\begin{array}{l}\text { A: inseto } \\
\text { vetor adulto }\end{array}$} & \multirow{3}{*}{$\begin{array}{l}\mathrm{A}_{1} \text { : estado } \\
\text { epidemiológico }\end{array}$} & 0: não infectivo \\
\hline & & 1: infectivo desde a fase de ninfa \\
\hline & & 2: infectivo a partir da fase adulta \\
\hline & $\begin{array}{l}\mathrm{A}_{2} \text { : localização } \\
\text { geográfica }\end{array}$ & $\left(\mathrm{x}_{\mathrm{a}}, \mathrm{y}_{\mathrm{a}}\right)$ : localização na área de plantio (sobre uma planta) \\
\hline
\end{tabular}


As premissas do MBI são dadas por:

- Características dos indivíduos: cada indivíduo é caracterizado por: idade (no caso das ninfas), estado epidemiológico, localização;

- Mudança de estado: uma vez em um estado epidemiológico, um indivíduo pode mudar o estado da doença em cada instante de tempo; de acordo com determinadas probabilidades;

- Distribuição de probabilidade: adotou-se a distribuição uniforme para as probabilidades de infecção, aquisição, reprodução e morte dos indivíduos;

- Processo de infecção: insetos vetores tornam-se infectivos quando estão localizados em hospedeiros infectados (assintomáticos ou sintomáticos), de acordo com determinadas probabilidades (Tabela 2). Da mesma forma, hospedeiros tornam-se infectados à partir da chegada de insetos vetores infectivos, segundo certa probabilidade (Tabela 2);

- Aspecto espacial: considera-se que a infecção de uma determinada planta sadia está relacionada com o estado das plantas vizinhas, uma vez que cada inseto vetor adulto possui uma probabilidade de visitar uma planta vizinha em cada iteração da simulação. Assume-se que as plantas estão distribuídas num reticulado de determinada área, com localização $(\mathrm{x}, \mathrm{y})$ no reticulado e vizinhança-4.

A dinâmica de interação entre as populações é determinada pelas regras de transição entre as categorias de indivíduos, que evoluem estocasticamente no tempo. A dinâmica do modelo é descrita a seguir, considerando os parâmetros listados na Tabela 2.

Tabela 2: Parâmetros biológicos do MBI. Fonte: Vilamiu et al [8].

\begin{tabular}{|c|c|c|}
\hline Parâmetro & Descrição & Valor \\
\hline$\tau_{\mathrm{h}}$ & Período de incubação da doença na planta & $6-18$ meses \\
\hline$\tau_{\mathrm{v}}$ & Tempo de duração da fase de ninfa & 15 dias \\
\hline$\mu_{\mathrm{v}}$ & Probabilidade de mortalidade de vetores adultos & $1 / 30$ \\
\hline$b$ & Probabilidade do inseto adulto visitar nova planta (por dia) & $1 / 15$ \\
\hline$\alpha$ & $\begin{array}{l}\text { Probabilidade de sucesso (produto da taxa de reprodução do } \\
\text { vetor adulto pela sobrevivência do vetor na faze de ninfa). }\end{array}$ & $1 / 30$ \\
\hline$\varepsilon$ & Probabilidade de detecção da doença no campo & 0.47 \\
\hline $\mathrm{p}^{\mathrm{a}}{ }_{\mathrm{v}}$ & $\begin{array}{l}\text { Probabilidade de infecção de plantas por vetores infectivos } \\
\text { na fase adulta }\end{array}$ & 0.01 \\
\hline $\mathrm{p}_{\mathrm{v}}^{\mathrm{n}}$ & $\begin{array}{l}\text { Probabilidade de infecção de plantas por vetores infectivos } \\
\text { desde a fase de ninfa }\end{array}$ & 0.67 \\
\hline $\mathrm{p}_{\mathrm{v}}$ & Probabilidade de aquisição da doença por insetos adultos & 0.13 \\
\hline $\mathrm{p}_{\mathrm{n}}$ & $\begin{array}{l}\text { Probabilidade de aquisição da doença por insetos na fase de } \\
\text { ninfa }\end{array}$ & 0.15 \\
\hline
\end{tabular}


- População N: a) se o indivíduo é não infectivo e atinge a idade $\tau_{\mathrm{v}}$, ele é transferido para a população A com estado epidemiológico $0 ;$ b) se o indivíduo é infectivo e atinge a idade $\tau_{\mathrm{v}}$, ele é transferido para a população A com estado epidemiológico 1 ; c) o indivíduo pode morrer com probabilidade $\mu_{\mathrm{n}}$; d) se o indivíduo for não infectivo e estiver localizado num hospedeiro infectado (estados epidemiológicos 1 à $\tau_{\mathrm{v}}$ ), ele pode se tornar infectivo com probabilidade $\mathrm{p}_{\mathrm{n}}$; e) o indivíduo tem sua idade acrescida de 1 dia;

- População A: a) o indivíduo pode se reproduzir com probabilidade $\alpha$, gerando um novo indivíduo da população A com idade $=0$ e estado não infectivo; b) se o indivíduo for não infectivo e estiver localizado num hospedeiro infectado, ele passa para o estado epidemiológico 2 (infectivo a partir da fase adulta ) com probabilidade $\mathrm{p}_{\mathrm{v}}$; c) o indivíduo se move com probabilidade $b$ para um dos seus hospedeiros vizinhos existentes (esquerda, direita, em cima, em baixo, escolhido aleatoriamente); d) o indivíduo pode morrer com probabilidade $\mu_{\mathrm{v}}$;

- População H: a) se o indivíduo for assintomático (estado epidemiológico $0<\mathrm{x}<\tau_{\mathrm{h}}$ ) é necessário acrescer 1 dia em $\mathrm{x}$; b) se o indivíduo for suscetível, ele pode alterar seu estado epidemiológico para assintomático $(\mathrm{x}=1)$ com probabilidade $\mathrm{p}_{\mathrm{v}}^{\mathrm{n}}$, considerando cada indivíduo da população A localizado em sua posição; c) se o indivíduo for suscetível, ele pode alterar seu estado epidemiológico para assintomático com probabilidade $\mathrm{p}_{\mathrm{v}}^{\mathrm{a}}$, considerando cada indivíduo da população $\mathrm{N}$ localizado em sua posição; d) se o indivíduo for sintomático, ele pode ser removido da área de plantio com probabilidade $\varepsilon$.

\section{Resultados}

O MBI descrito acima foi implementado utilizando o software Matlab. Para o início das simulações considerou-se uma população de 400 plantas sadias distribuídas num reticulado $20 \times 20$ e a chegada de 4.000 insetos vetores pela lateral esquerda do reticulado, considerando que 5\% desses vetores adultos sejam infectivos desde a fase de ninfa.

Na Figura 1 temos a evolução espacial da doença na área, onde temos representado o estado epidemiológico do hospedeiro em três instantes: 1, 2 e 3 meses.
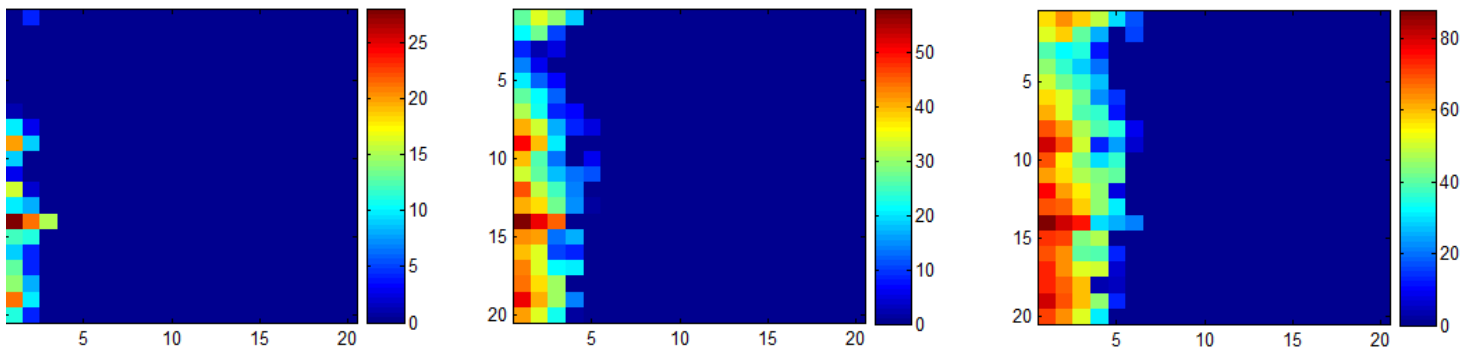

Figura 1: Estado epidemiológico do pomar constituído de uma área quadrada 20x20 com 400 hospedeiros, onde a infestação ocorre à partir da lateral esquerda. 

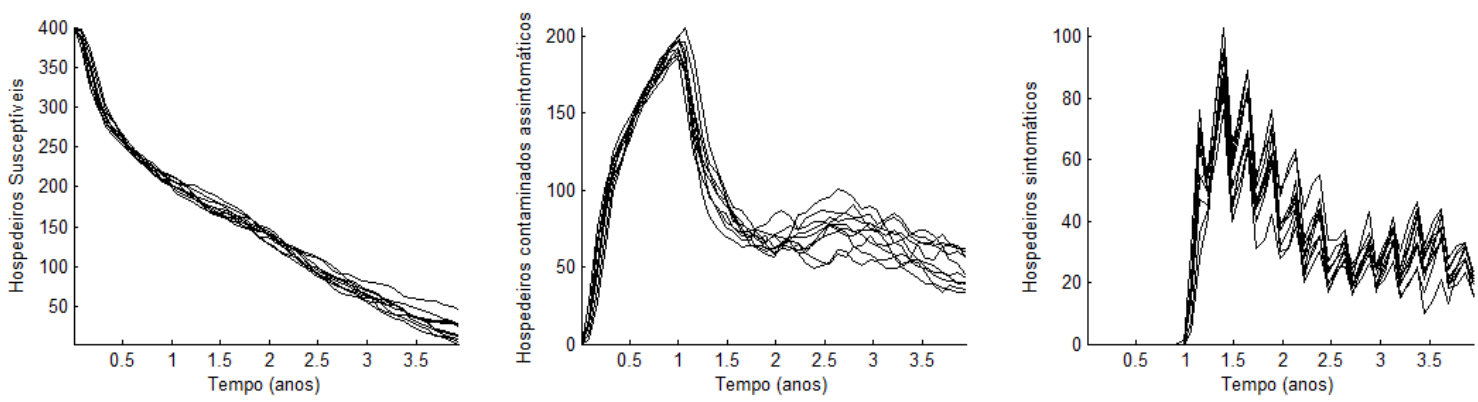

Figura 2: Dez simulações para a dinâmica da população de hospedeiros, separados segundo seu estado epidemiológico: susceptível (esquerda), contaminado assintomático (centro) e contaminado sintomático (direita).

Na Figura 2, temos o número de indivíduos em cada estado ao longo de um período de 4 anos. Por ela, vemos que mesmo com a remoção de plantas infectadas, este procedimento não consegue deter a doença dado o grande número de hospedeiros em período de incubação (não expressão sintomas).

Finalmente, na Figura 3, avaliamos um possível aumento na eficiência da estratégia de remoção de hospedeiros infectados considerando dois períodos de remoção. Em ambas os gráficos foram realizadas 100 simulações, sendo que no da esquerda foram usados os mesmos parâmetros apresentados na Tabela 2 e no da direita, o tempo de remoção utilizado foi reduzido para apenas 1 mês. Percebemos pela comparação entre os dois que o grande investimento necessário para aumentar a frequência das remoções não surtirá o efeito desejado.
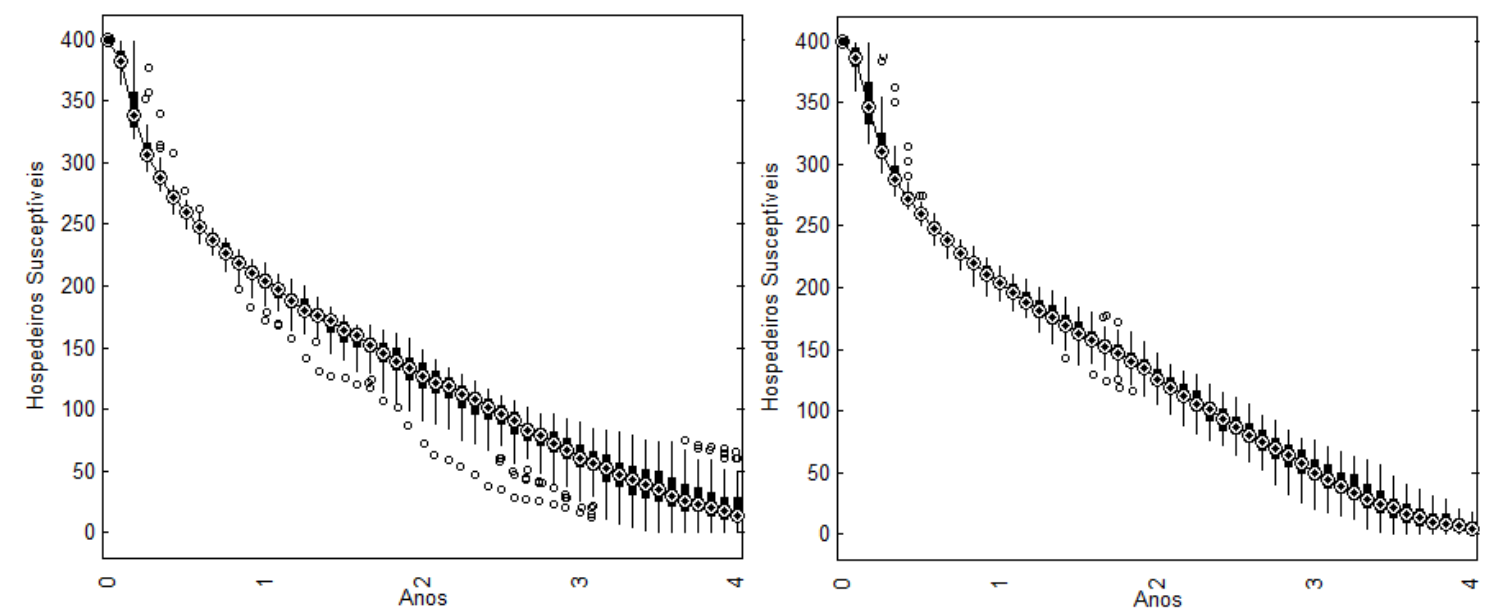

Figura 3: No gráfico da esquerda temos o gráfico boxplot, obtido à partir de 100 simulações do MBI utilizando os valores dos parâmetros presentes na Tabela 2. Na direita, o mesmo processo foi realizado mas, o período entre remoções de plantas sintomáticas foi reduzido de 3 meses para apenas 1 mês.

\section{Conclusões}

Mesmo com uma alta frequência (a cada mês) de remoção de plantas sintomáticas, o alastramento da doença na plantação acaba por contaminar todas as plantas. Assim, fica claro que outras estratégias precisam ser utilizadas para o controle da doença. Resultado semelhante foi alcançado em Vilamiu et al [8]. 


\section{Referências}

[1] Instituto Brasileiro de Geografia e Estatística, Banco de dados agregado, URL: $<$ www.sidra.ibge.gov.br/bda/tabela/listabl.asp?c=1613\&z=t\&o=11 >, 2013.

[2] M. F. Neves, F. F. Lopes, V. G. Trombin, A. A. Amaro, E. M. Neves, and M. S. Jank. "Caminhos para a citricultura - Uma Agenda para Manter a Liderança Mundial", Editora Atlas, 2007.

[3] Ministério da Agricultura, Pecuária e Abastecimento. Assessoria de Gestão Estratégica, Projeções do agronegócio: Brasil 2012/2013 a 2022/2023, 2013.

[4] J. M. Bové. Huanglongbong: a destructive, newly-emerging, century-old disease of citrus. Journal of Plant Pathology 88, 7-37, 2006.

[5] L. M. Contreras-Medina, I. Torres-Pacheco, R. G. Guevara-González, R. J. RomeroTroncoso, I. R. Terol-Villalobos, and R. A. Osornio-Rios, Mathematical modeling tendencies in plant pathology. African Journal of Biotechnology 8, 7399 - 7408, 2009.

[6] R. A. C. Jones, M. U. Salam, T. J. Maling, A. J. Diggle, and T. D. J. Principles of predicting epidemics of plant virus disease. Annual Review of Phytopathology 48, 179 203, 2010.

[7] M. J. Jeger, J. Holt, F. van den Bosch, and L. V. Madden. Epidemiology of insecttransmitted plant viruses: modelling disease dynamics and control interventions. Physiological Entomology 29, 291 - 304, 2004.

[8] R. G. d'A. Vilamiu, S. Ternes, G. A. Braga, and F. F. Laranjeira, A model for Huanglongbing spread between citrus plants including delay times and human intervention. Doi:10.1063/1.4756657. 2012.

[9] Giacomini, H.C. Sete Motivações Teóricas para o Uso da Modelagem Baseada no Indivíduo em Ecologia. Acta Amazonica, 37(3), 431-446. 2007.

[10] Nepomuceno, E.G. "Dinâmica, Modelagem e Controle de Epidemias", Tese de Doutorado, UFMG, 2005. 